other men who have sex with men (MSM). In BC, all syphilis cases and partner notification (PN) are managed centrally. We describe trends in PN outcomes from 2010-2013.

Methods We conducted chart reviews on a random sample $(\mathrm{n}=$ $350)$ representing $33 \%(350 / 1054)$ of all IS cases among MSM in BC from 2010-2013. We described trends in number of anonymous and notifiable partners, proportions notified and tested, and test outcomes.

Results The 350 cases (44\% primary/secondary, 56\% early latent) reported 1942 partners; 1131 (58\%, range 46-67\%) partners were notifiable. From 2010-2013, the number of partners per case increased (average 5 to $8 ; 6 \%$ to $16 \%$ reported $\geq 10$ partners). Of the 1131 notifiable partners, 936 (83\%) were notified: $638(56 \%)$ by the case, $252(22 \%)$ by a healthcare provider (HCP), and $46(4 \%)$ by other means (e.g., referred to another jurisdiction). From 2010-2013, the proportion of casenotified partners was stable while the proportion of HCP-notified partners decreased ( $43 \%$ to $19 \%)$. Of the 936 partners notified, $287(31 \%)$ were known to be tested (21\% [133/638] of case-notified and 54\% [135/252] of HCP-notified partners). Of these, $62(22 \%)$ partners tested positive and were treated. From 2010-2013, the proportion of partners known to be tested decreased, primarily due to a decrease among case-notified partners $(46 \%$ to $15 \%)$.

Conclusion Overall $83 \%$ of notifiable partners were notified. Challenges to PN among MSM in BC include anonymous partners, the increasing number of partners to be notified, and documentation of outcomes for case-notified partners. While selfnotification appears to be preferred by many MSM, partner testing may be lower in this group. Strategies to encourage partner testing (whether case or HCP-notified) are needed and may be best focused on MSM with more partners.

Disclosure of interest statement The BC Centre for Disease Control is a provincial public health agency which has a responsibility for surveillance of communicable diseases, including HIV and sexually transmitted infections. No external funding was received for this study. All authors have no conflicts of interest.

\section{P04.07 "SUMMER LOVING": AN ANALYSIS OF SEASONAL DIFFERENCES IN SEXUAL BEHAVIOUR AND SEXUALLY TRANSMISSIBLE INFECTIONS}

1,2VJ Cornelisse* ${ }^{*}, 2$ CK Fairley, ${ }^{1,2}$ EPF Chow. ${ }^{1}$ Central Clinical School, Faculty of Medicine, Nursing and Health Sciences, Monash University, Melbourne, Victoria, Australia; ${ }^{2}$ Melbourne Sexual Health Centre, Alfred Health, Carlton, Victoria, Australia

\subsection{6/sextrans-2015-052270.261}

Introduction Public health campaigns must be timed to target their audience at times of highest risk. To date no study has correlated seasonal differences in sexual behaviour with those in sexually transmissible infection (STI) positivity; and no seasonal study has been conducted in the southern hemisphere.

Our study aimed to describe seasonal differences in sexual behaviour and correlate this with STI positivity.

Methods This was a cross-sectional study of individuals attending the Melbourne Sexual Health Centre (MSHC) over a 9 year period from 2006-2014 inclusive. We conducted separate analyses for women, men who have sex with men (MSM) and men who have sex with women (MSW). Seasonal patterns of sexual behaviour (partner numbers, condom use and injecting drug use), and STI positivity were examined in all groups. STI with a high symptomatic rate and short incubation period were selected for the analysis: gonorrhoea, primary herpes simplex virus
(HSV) infections, non-gonococcal urethritis (NGU) (men only), and pelvic inflammatory disease (PID) (women only).

Results All groups reported a significantly higher number of partners in summer compared to winter (women $\mathrm{P}<0.001$; MSW P $<0.001$; MSM P $=0.004)$.

MSW reported less consistent condom use in summer ( $\mathrm{P}=$ 0.016); a similar but non-significant trend was observed in MSM and women.

The urethral gonorrhoea positivity among MSM was significantly higher in summer compared to winter $(\mathrm{P}=0.017)$. Similarly, the NGU positivity among MSW was the highest in summer $(\mathrm{P}=0.009)$.

In women PID diagnoses climbed over summer to peak in autumn, which then dropped to a low in winter $(\mathrm{P}=0.025)$.

The other STIs did not show statistically significant seasonal differences.

Conclusion Our study describes a peak in sexual partner number and STI positivity in summer. This seasonal difference must inform the timing of public health campaigns, as these are likely to be maximally effective in spring and summer.

Disclosure of interest statement None.

\section{P04.08 SEX PARTNER MEETING VENUES AND HIV TRANSMISSION RISK FACTORS AMONG NEWLY DIAGNOSED HIV-INFECTED URBAN MSM: IMPLICATIONS FOR TARGETED CONTROL STRATEGIES}

${ }^{1,2}{ }^{E L}$ Fields ${ }^{*},{ }^{3} \mathrm{MA}$ Clarke, ${ }^{1,4} \mathrm{C}$ Schumacher, ${ }^{1} \mathrm{AG}$ Safi, ${ }^{4} \mathrm{C}$ Nganga-Good, ${ }^{4} \mathrm{R}$ Muvva, ${ }^{4,5,6 \mathrm{P}}$ Patrick Chaulk, ${ }^{1,3} \mathrm{JM}$ Jennings. ${ }^{1}$ Center for Child and Community Health Research, Department of Pediatrics, Johns Hopkins School of Medicine, Baltimore, MD, USA; ${ }^{2}$ Department of Health Behavior and Society, Johns Hopkins Bloomberg School of Public Health, Baltimore, MD, USA; ${ }^{3}$ Department of Epidemiology, John Hopkins Bloomberg School of Public Health, Baltimore, MD, USA; ${ }^{4}$ Baltimore City Health Department, Baltimore, MD, USA; ${ }^{5}$ Department of Medicine, Johns Hopkins School of Medicine, Baltimore, MD, USA; ${ }^{6}$ Department of Health Policy and Management, John Hopkins Bloomberg School of Public Health, Baltimore, MD, USA

10.1136/sextrans-2015-052270.262

Background Men who have sex with men (MSM) experience over half of US HIV infections. Control strategies, including identifying venues with ongoing transmission, are needed. Surveillance efforts traditionally focus on place, rather than internet venues and may miss other high risk populations. The objective of this study was to determine if there were significant differences in HIV-transmission related risk factors including viral load by sex partner meeting venue among newly diagnosed HIVinfected MSM.

Methods Public health surveillance data of newly diagnosed MSM between January 2011 and July 2014 ( $\mathrm{N}=280$ ) was utilised. Data included reported past-year sex partner meeting venues and transmission risk factors including viral load collected between October 2012 and July 2014.

Results Eighty-four percent of subjects were Black and $48 \%$ were $\leq 24$ years-old; $39 \%$ reported meeting partners at place only venues (bar/club/park/street), 34\% internet only venues (website/mobile-app), and $26 \%$ both venue types. Significant differences among the three groups of individuals included differences in reports of sex under the influence of alcohol, numbers of sex partners and chlamydia co-infection at time of HIV diagnosis; individuals reporting both venue types had higher reports in all three characteristics compared to individuals reporting place only and venue only. Individuals reporting both venue types had significantly lower geometric mean viral load (copies/ 